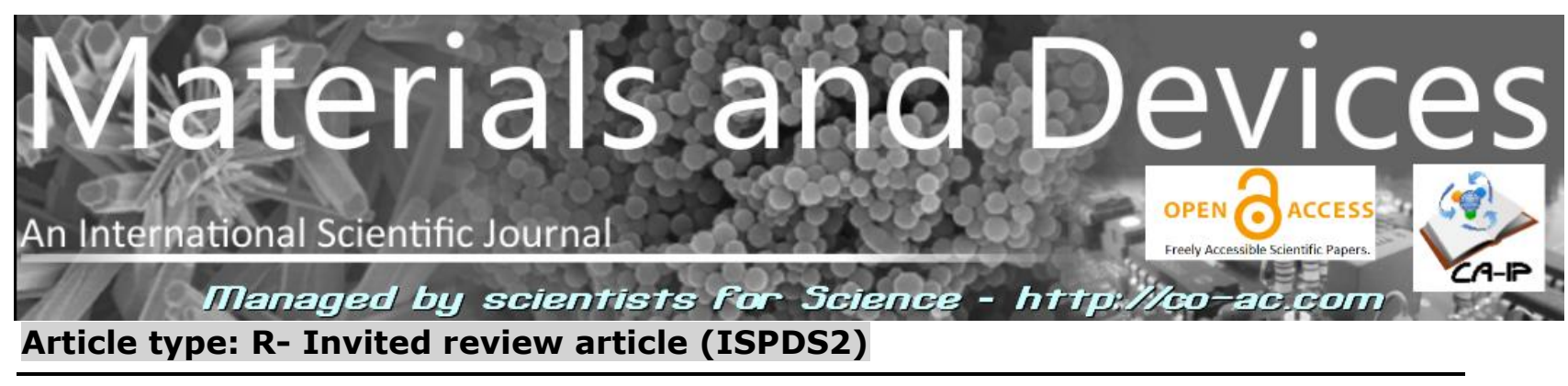

\title{
Lecture on the anomalous diffusion in Condensed Matter Physics
}

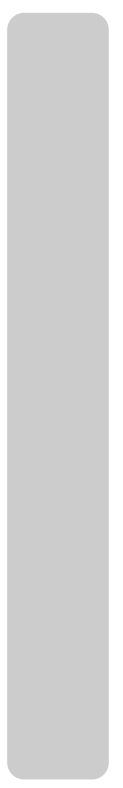

M. Benhamou

Physics Department, Faculty of Sciences, Moulay Ismail University,

m.benhamou@ensam-umi.ac.ma

RECEIVED: 9 July 2018 / RECEIVED IN FINAL FORM: 28 July 2018 / ACCEPTED: 31 July 2018

\begin{abstract}
Diffusion is a natural or artificial process that governs many phenomena in nature. The most known diffusion is the Brownian or normal motion, where the mean-square-displacement of the tracer (diffusive particle among others) increases as the square-root of time. It is not the case, however, for complex systems, where the diffusion is rather slow, because at small-scales, these media present an heterogenous structure. This kind of slow motion is called subdiffusion, where the associated mean-square-displacement increases in time, with a non trivial exponent, $\alpha$, whose value is between 0 and 1 . In this review paper, we report on new trends dealt with the study of the anomalous diffusion in Condensed Matter Physics. The study is achieved using a theoretical approach that is based on a Generalized Langevin Equation. As particular crowded systems, we choose the so-called Pickering emulsions (oil-in-water), and we are interested in how the dispersed droplets (protected by small solid charged nanoparticles) can diffuse in the continuous phase (water). Dynamic study is accomplished through the mean-square-displacement and the velocity-autocorrelation-function. Finally, a comparison with Molecular Dynamics data is made.
\end{abstract}

Keywords: COMPLEX SYSTEMS, ANOMALOUS DIFFUSION, GENERALIZED LANGEVIN EQUATIONS, MEAN-SQUARE-DISPLACEMENT, VELOCITY-AUTOCORRELATION-FUNCTION, MOLECULAR DYNAMICS SIMULATION.

\section{Introduction}

The anomalous diffusion in various fields of science (physics, chemistry, biology, ecology...) has received much attention from a theoretical and experimental point of view. In fact, such a diffusion 
appears in heterogeneous, disordered, fractal, colloidal and polymer systems, and in general, it produces within the complex systems containing entities that move on different scale-times. In opposition to the normal or Brownian diffusion, the anomalous diffusion is slower, due to an extreme difficulty that a particle (tracer) moves in a complex structure. This kind of diffusion is usually referred to as subdiffusion, and it is characterized by a mean-square-displacement (MSD) that behaves as: $\mathrm{W}(\mathrm{t}) \equiv\left\langle(\mathbf{x}(\mathrm{t})-\mathbf{x}(0))^{2}\right\rangle=2 \mathrm{D}_{\alpha} \mathrm{t}^{\alpha}$, with $0<\alpha<1$. This large-time behavior then deviates from the linear dependence on time found for the Brownian motion ${ }^{[1-3]}$. In expression above, $\mathbf{x}(t)$, represents the time-position of the random walker, and the generalized diffusion coefficient, $\mathrm{D}_{\alpha}$, also called "fractional diffusion coefficient", is expressed in length ${ }^{2} /$ time $^{\alpha}$ unit.

We emphasize that the subdiffusion is a feature of the crowded systems, where the trajectories of their mobile constituents are strongly correlated. Notice that the above scaling relation is valid for large-times, that is beyond some characteristic time depending on the specific details of the diffusion process and the structure of the host medium. In general, a particle is said to be subdiffusive if the condition $\mathrm{W}(\mathrm{t}) / \mathrm{t} \rightarrow 0$, for $\mathrm{t} \rightarrow+\infty$, is satisfied (very slow diffusion). This explains why the anomalous exponent, $\alpha$, must be in the interval $0<\alpha<1$.

It is noted that the subdiffusive transport appears in a variety of systems, such as the random-walk in fractal structures ${ }^{[2]}$, fractional-time Brownian motion ${ }^{[4]}$, living systems ${ }^{[5]}$, charge carrier transport in amorphous semiconductors ${ }^{[6,7]}$, NMR diffusometry on percolation structures ${ }^{[8]}$, and the motion of a colloid in a polymer network ${ }^{[9]}$. For example, for diffusion in fractal structures, $\alpha=2 / d_{w}$, where $\mathrm{d}_{\mathrm{w}}>2$ is the walk-dimension $\left(\mathrm{d}_{\mathrm{w}}=2 \mathrm{~d}_{\mathrm{f}} / \mathrm{d}_{\mathrm{s}}\right.$, where $\mathrm{d}_{\mathrm{f}}$ and $\mathrm{d}_{\mathrm{s}}$ are the fractal and spectral dimensions, respectively), and for the fractional-time Brownian motion, $\alpha=2 \mathrm{H}$, where $H$ is the Hurst index. Examples of enhanced diffusion $(\alpha>1)$ include tracer particles in vortex arrays in a rotating flow ${ }^{[10]}$, layered velocity fields ${ }^{[11]}$, and Richardson diffusion ${ }^{[12]}$. The case $1<\alpha<2$ refers to superdiffusion (turbulent plasmas, Levy-flights, transport in polymers), $\alpha=2$, to ballistic diffusion (optical traps), and $\alpha=3$, to Richardson diffusion (atmospheric turbulence). The subdiffusion or superdiffusion exponent, $\alpha$, is not a universal quantity, but mainly depends on the pertinent parameters that control the phenomenon. As we shall see below, for the subdiffusion of the clothed droplets of Pickering emulsions, this exponent shall depend on their size, density and surface charge, and the saltconcentration.

Anomalous diffusion is a subject which has been extensively studied, especially in the past decade by using the so-called Generalized Langevin Equation (GLE) (with memory) ${ }^{[13,14-19]}$. The last has been introduced by Kubo ${ }^{[20]}$, as a generalization of the standard Langevin equation describing the Brownian or normal diffusion. Another useful approach to the anomalous diffusion is based on the fractional diffusion equation ${ }^{[21-23]}$, fractional Fokker-Planck equation ${ }^{[21,24]}$, generalized ChapmanKolmogorov equation ${ }^{[25]}$ and fractional generalized Langevin equation ${ }^{[26,27]}$.

In their seminal paper, Mainardi and Pironi ${ }^{[28]}$ had introduced a fractional Langevin equation taking into account the retarding effects due to hydrodynamic backflow and showed that it is a particular case of GLE. These equations can be analyzed by using the properties and asymptotic behaviors of Mittag-Leffler (M-L) functions ${ }^{[29-41]}$.

For the reader, an exhaustive list of other important developments of the subject can be found in Ref. [42].

In this paper, we show how GLE can be applied to study the sub-diffusion phenomenon within particular complex systems which are Pickering emulsions (PEs) ${ }^{[43,44]}$, and compare results from this equation to Molecular Dynamics (MD) data ${ }^{[45]}$. This computational method was introduced by Alder and Wainwright ${ }^{[46]}$, in order to study the structure and dynamics of liquids.

PEs are dispersions presenting, very often, as oil-in-water $(\mathrm{O} / \mathrm{W})$, water-in-oil $(\mathrm{W} / \mathrm{O})$ or double emulsion water-oil-water $(\mathrm{W} / \mathrm{O} / \mathrm{W})$. These dispersions are stabilized by an addition of small solid (organic or inorganic) particles that act as emulsifiers, instead of the surfactant molecules ${ }^{\text {[47-50]. These }}$ particles that act as emulsifiers are of nanometric size, while the stabilized droplets are as small as few micrometers diameter. The stabilization of larger droplets (few millimeters diameter) is possible as well, using micron-sized solid particles. The stabilization of the dispersed droplets within PEs is ensured by a strong adsorption of the solid nanoparticles at their surfaces. In contrary to surfactants, where the adsorption is rather dynamic (reversible), that of the charged solidparticles is irreversible and sufficiently strong, with a very high adsorption energy, between $\mathrm{k}_{\mathrm{B}} \mathrm{T}$ and $10^{8} \mathrm{k}_{\mathrm{B}} \mathrm{T}$, where $\mathrm{k}_{\mathrm{B}} \mathrm{T}$ is the thermal energy. Here, $\mathrm{k}_{\mathrm{B}}$ is the Boltzmann's constant and $\mathrm{T}$ is the bath temperature. Of course, such an energy mainly depends on the value of the wetting (or contact) angle and the droplet-radius ${ }^{[50]}$. 
The anchoring of the solid particles emanates from a partial wetting of the surface of the charged solid particles by water and oil.

Due to their remarkable properties, such as high stability with respect to coalescence and their recent use in nanotechnology leading to the creation and the characterization of the nano-scale structures in new ways, PEs have been the subject of many studies, both from experimental and theoretical point of views. In addition, they can serve as templates for the advanced materials ${ }^{[51,52]}$, as Janus colloids ${ }^{[53]}$, composite particles ${ }^{[54-61]}$ and colloidosomes ${ }^{[62-64]}$.

PEs are heterogenous liquids which present as a dispersion of droplets of some liquid (dispersed phase) in another one (continuous phase). The two liquids are not chemically and physically compatible. By a mechanical mixing of the emulsion, each droplet becomes surrounded by discrete nanoparticles arranged on its surface. For the study of PEs, the clothed droplets can be regarded as charged soft-colloids ${ }^{[65]}$. These charged droplets are subject to an anomalous diffusion as observed by MD simulations ${ }^{[45]}$, and we are interested in how they really move using a GLE approach. The dynamics of these droplets are studied through two main quantities, which are MSD and the velocityautocorrelation-function (VACF). As we shall see below, for an appropriate choice of the memoryfunction, the solutions to GLE express themselves in terms of $M-L$ functions.

The remaining of the presentation proceeds as follows. In the first section, we present a general dynamic theory, based on a GLE. A comparison between the results from this theory with MD data is the aim of the third section. Finally, some concluding remarks are drawn in the last section.

\section{Dynamic theory}

Consider a given particle, termed tracer or random walker, which executes a diffusion movement in the host medium. The raised question is how this random walker diffuses in the host medium, beyond some characteristic time we will precise below. In this time-regime, the random walker (target) feels to be trapped in a cage formed by the surrounding particles (traps), and then, it cannot escape from this cage, except after a long-time. We denote by $\mathrm{N}_{\mathrm{c}}$, the average-number of traps around the considered random walker. Consequently, the presence of the traps makes difficult such a diffusion process, and then, the random walker executes rather a subdiffusion, characterized by an anomalous exponent, $\alpha$. The latter depends essentially on the nature of the random process and that of the host medium.

The basic dynamic quantities of our interest are MSD, $\left\langle(\mathbf{x}(\mathrm{t})-\mathbf{x}(0))^{2}\right\rangle \equiv \mathrm{W}(\mathrm{t})$, and VACF, $\langle\mathbf{v}(\mathrm{t}) \cdot \mathbf{v}(0)\rangle \equiv \mathrm{c}_{\mathrm{vv}}(\mathrm{t})$. They are related by

$$
W(t)=2 \int_{t_{0}}^{t} d t^{\prime}\left(t-t^{\prime}\right) c_{v v}\left(t^{\prime}\right), \quad c_{v v}(t)=\frac{1}{2} \frac{d^{2} w(t)}{d t^{2}}
$$

To investigate quantitatively the cage effect and the subdiffusion phenomenon, the starting point is a GLE developed in the past by Kubo ${ }^{[20]}$ and by Zwanzig ${ }^{[66]}$. Then, GLE describing the motion of the random walker reads

$$
\mathrm{m} \frac{\mathrm{d} v(\mathrm{t})}{\mathrm{dt}}=-\mathrm{m} \int_{0}^{\mathrm{t}} \mathrm{dt} \mathrm{t}^{\prime} \gamma\left(\mathrm{t}-\mathrm{t}^{\prime}\right) \mathbf{v}\left(\mathrm{t}^{\prime}\right)+\xi(\mathrm{t})
$$

Here, $\gamma(\mathrm{t})$ is the memory-function that expresses a retardation to friction. The random force, $\xi(t)$, satisfies

$$
\langle\xi(t)\rangle=\mathbf{0}, \quad\langle\xi(t) \cdot \xi(0)\rangle=m_{B} T \gamma(t)
$$

For viscous colloidal solutions, the particle acceleration vanishes, and in this case, GLE equation reads

$$
-m v_{0} \delta(t)=-m \int_{0}^{t} d t^{\prime} \gamma\left(t-t^{\prime}\right) \mathbf{v}\left(t^{\prime}\right)+\xi(t)
$$

where $\mathrm{v}_{0}=\left|\mathrm{v}_{0}\right|=\sqrt{2 \mathrm{k}_{\mathrm{B}} \mathrm{T} / \mathrm{m}}$ is the initial velocity of the tracer and the stochastic force, $\xi(\mathrm{t})$, also satisfies relations (3). 
Assume, now, that the memory-function has the form

$$
\gamma(\mathrm{t})=\gamma_{0} \delta(\mathrm{t})+\frac{\gamma_{\alpha}}{\Gamma(1-\alpha)} \mathrm{t}^{-\alpha} \quad(0<\alpha<1)
$$

Here, $\gamma_{0}$ and $\gamma_{\alpha}$ are two positive constants, $\Gamma(\mathrm{x})$ is the Euler gamma function and $\delta(\mathrm{t})$ represents the Dirac-distribution. Using the Laplace-transform techniques together with relations (3), we find that MSD and VACF, in the non-inertial regime, are given by

$$
\begin{gathered}
\mathrm{W}(\mathrm{t})=2 \frac{\mathrm{k}_{\mathrm{B}} \mathrm{T}}{\mathrm{m} \gamma_{0}} \mathrm{tE}_{1-\alpha, 2}\left(-\left(\frac{\mathrm{t}}{\tau}\right)^{1-\alpha}\right) \\
\mathrm{c}_{\mathrm{vV}}(\mathrm{t})=\frac{\mathrm{k}_{\mathrm{B}} \mathrm{T}}{\mathrm{m} \gamma_{0}} \frac{\mathrm{d}^{2}}{\mathrm{~d}^{2}}\left[\mathrm{tE}_{1-\alpha, 2}\left(-\left(\frac{\mathrm{t}}{\tau}\right)^{1-\alpha}\right) \theta(\mathrm{t})\right]
\end{gathered}
$$

with the Heaviside step-function, $\theta(\mathrm{x})$. In these expressions, $\mathrm{E}_{\sigma, \tau}(\mathrm{x})$ denotes the twoparameter $M-L$ function ${ }^{[29]}$. There, $\tau=\left(\gamma_{0} / \gamma_{\alpha}\right)^{1 /(1-\alpha)}$ is a time-scale.

For small-times, in comparison with the characteristic one, $\tau$, MSD and VACF behave, respectively, as

$$
\begin{array}{ll}
\mathrm{W}(\mathrm{t})=2 \mathrm{D}_{0} \mathrm{t} & (\mathrm{t} \ll \tau) \\
\mathrm{c}_{\mathrm{vv}}(\mathrm{t})=\mathrm{D}_{0} \delta(\mathrm{t}) & (\mathrm{t} \ll \tau)
\end{array}
$$

Then, at these time-scales, the random walker executes a Brownian (normal) diffusion, with the usual diffusion coefficient, $\mathrm{D}_{0}=\mathrm{k}_{\mathrm{B}} \mathrm{T} / \mathrm{m} \gamma_{0}$. For large-times, compared to $\tau$, we find

$$
\begin{array}{crl}
\mathrm{W}(\mathrm{t}) & =2 \mathrm{D}_{\alpha} \mathrm{t}^{\alpha} & (t \gg \tau) \\
\mathrm{c}_{\mathrm{Vv}}(\mathrm{t})=\alpha(\alpha-1) \mathrm{D}_{\alpha} \mathrm{t}^{\alpha} & (\mathrm{t} \gg \tau)
\end{array}
$$

with

$$
D_{\alpha}=\frac{D_{0}}{\Gamma(\alpha+1)}
$$

which denotes the generalized diffusion coefficient.

\section{Comparison with MD simulations}

For the description of the dynamic properties of PEs, using MD method, the equations of motion are solved in the canonical ensemble using the Velocity Verlet Algorithm (VVA) ${ }^{[67]}$, with the Thermostat of Berendsen ${ }^{[68]}$, in order to keep the temperature constant. In addition, periodic boundary conditions are applied to remove the surface effects and simulate an infinite emulsion. In the following, it will be convenient to use dimensionless units, where the length unit is $\sigma$, time in units of $\tau=\sigma \sqrt{\mathrm{M} / \varepsilon}$, where $\mathrm{M}$ is the oil-droplet mass and $\varepsilon$ is the depth of the interaction potential, $\mathrm{k}_{\mathrm{B}} \mathrm{T}$ is the energy unit, and $\mathrm{L}_{0}=\mathrm{N} \times(6 \mathrm{~V} / \pi \mathrm{N})^{1 / 3}$ is the box-size, where $\mathrm{N}$ is the number of the clothed oil-droplets, and $\mathrm{V}$ is the volume of simulation box (in periodic conditions). MD simulations ${ }^{[45]}$ where carried out with 1728 oildroplets and the dimensionless time-step for VVA is chosen to be 0.05 . The last typical value of steptime is that usually adopted in studying the colloidal solutions from MD simulations.

Discussion on dynamics of PEs was made ${ }^{[45]}$ varying the pertinent factors, as the surface charge of oil-droplets, and their density and the concentration of the added salt. 


\section{Charge effects}

The first goal was the quantification of the influence of the charge carried by the oil-droplets on their dynamic properties ${ }^{[45]}$. The size of the oil-droplets, their reduced number density and the saltconcentration were fixed to the values: $\sigma=20000 \AA, \rho^{*}=0.0020$ and $c_{s}=2.91 \mu \mathrm{M}$. But the valence, $\mathrm{Z}$, was varied from 1000 to 4500 .

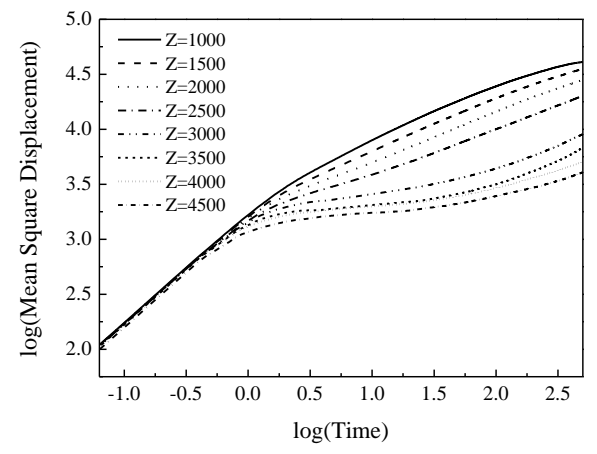

Figure 1: MSD versus dimensionless time, $t / \tau$, in log-log scale, for various values of the oil-droplet surface charge, with the fixed parameters: $\sigma=20000 \AA, \rho^{*}=0.0020$ and $c_{s}=2.91 \mu \mathrm{M}$.

Figure 1 shows the log-log plot of the reduced MSD upon dimensionless time, $t / \mathrm{t}$, for various values of the surface charge. Such a figure clearly shows the existence of two time-regimes. For early times, the random walker executes a normal diffusion, independently of the value of the surface charge. This agrees with the theoretical behavior, relation (8). Above some known characteristic time, it executes rather a subdiffusion, and MSD behaves as $W(t) \sim D_{\alpha} t^{\alpha}$, with an average subdiffusion exponent $\alpha_{c}=0.418$. This result is in perfect agreement with the theoretical behavior, relation (10). As already shown ${ }^{[45]}$, both anomalous diffusion exponent, $\alpha$, and fractional diffusion coefficient, $D_{\alpha}$, decrease progressively with the surface charge. As the later is augmented, the correlations between the oildroplets become stronger, and consequently, one assists to a slow dynamic.

Figure 2 indicates the time-variation of VACF, for the same values of the surface charge. The abrupt increase of this function, at small-times, can be explained by formula (9) replacing the Diracdistribution $\delta(t)$ by a very narrow Gaussion function, that is: $\delta(t) \rightarrow(2 \pi \sigma)^{-1 / 2} \exp \left(-t^{2} / 2 \sigma\right)$, with a very small time-scale $\sigma$. For large-times, VACF goes to zero, in agreement with theoretical formula, relation (11).

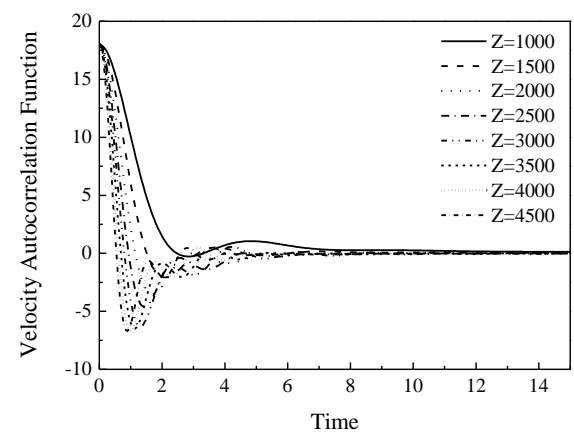

Figure 2: VACF versus dimensionless time, $t / \tau$, in log-log scale, for various values of the oil-droplet surface charge, with the fixed parameters: $\sigma=20000 \AA, \rho^{*}=0.0020$ and $c_{s}=2.91 \mu \mathrm{M}$.

\section{Density effects}

The second goal was the study of the influence of the density of the clothed oil-droplets on their dynamics ${ }^{[45]}$, fixing their surface charge and size, and the salt-concentration to the values: $\mathrm{Z}=$ 
$2000, \sigma=20000 \AA$ and $\mathrm{c}_{\mathrm{s}}=2.91 \mu \mathrm{M}$.

Figure 3 shows the log-log plot of MSD, versus dimensionless time, $t / \tau$, for various values of the droplet-density. As above, this MSD exhibits two time-regimes: normal, with a density-dependent diffusion coefficient, and subdiffusive, with an average subdiffusion exponent, $\alpha_{d}=0.432$. This is in good agreement with the theoretical formulae (8) and (10).

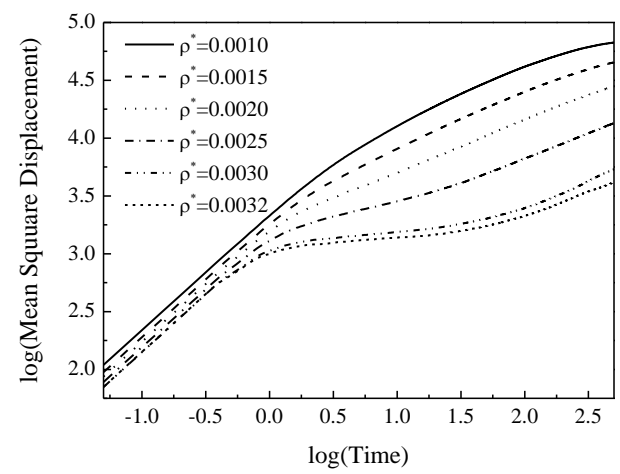

Figure 3: MSD versus dimensionless time, $t / \tau$, in log-log scale, for various values of the oil-droplet density, with the fixed parameters: $\mathrm{Z}=2000, \sigma=20000 \AA$ and $\mathrm{c}_{s}=2.91 \mu \mathrm{M}$.

Figure 4 indicates the variation of VACF upon time, for the same parameters as before. The timebehavior of this dynamic property agrees well with the theoretical predictions, relations (9) and (11).

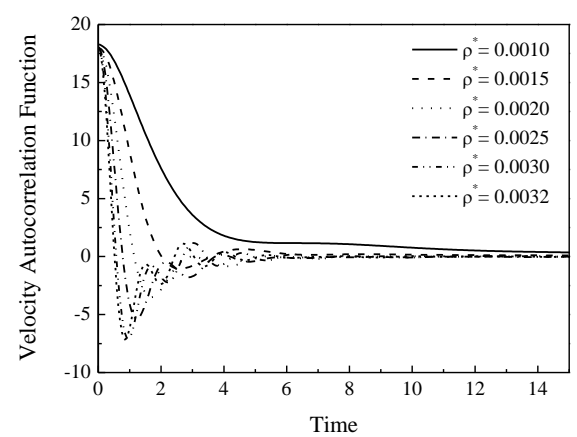

Figure 4: VACF versus dimensionless time, $t / \tau$, in log-log scale, for various values of the oil-droplet density, with the fixed parameters: $\mathrm{Z}=2000, \sigma=20000 \AA$ and $\mathrm{c}_{\mathrm{s}}=2.91 \mu \mathrm{M}$.

\section{Salt-concentration effects}

A quantitative investigation of the effects of the salt-concentration, $c_{s}$, on the oil-droplets dynamics was achieved in Ref. [45], fixing the remaining parameters to the values: $Z=500, \sigma=5000 \AA$, and $\rho^{*}=0.0020$. 


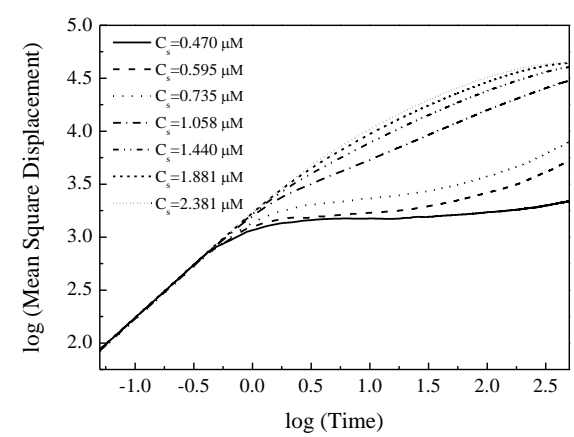

Figure 5: MSD versus dimensionless time, $t / \tau$, in log-log scale, for various values of the saltconcentration, with fixed parameters: $Z=500, \sigma=5000 \AA$ and $\rho^{*}=0.0020$.

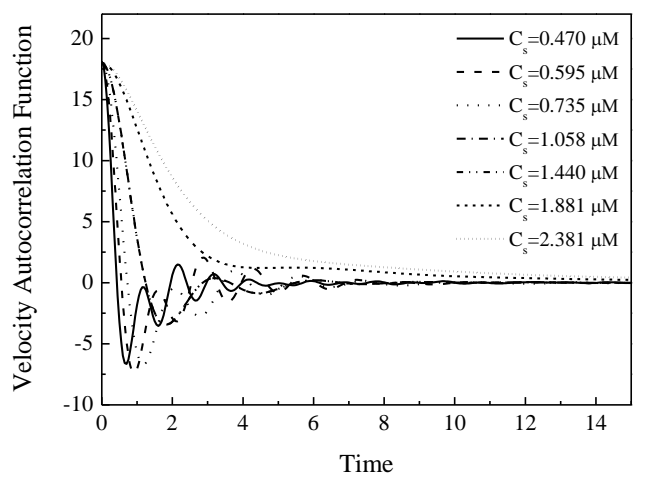

Figure 6: VACF versus the dimensionless time, $t / \tau$, in log-log scale, for various values of the saltconcentration, with fixed parameters: $Z=500, \sigma=5000 \AA$ and $\rho^{*}=0.0020$.

Figures 5 and 6 show the time-variation of MSD and VACF, respectively. Also, in this case, MD data are in good agreement with theory, relations (8) to (11). In particular, at large-time, MSD exhibits a subdiffuse behavior with an average exponent $\alpha_{s}=0.467$.

\section{Conclusion}

In this presentation, we focus on a quantitative dynamic study of the subdiffusion phenomenon within crowded systems, as Pickerings emulsions, which are stabilized by a strong irreversible adsorption of charged solid nanoparticles on the surface of the dispersed oil-droplets.

As we have shown above, the subdiffusion with complex systems may be approched well using a non-inertial generalized Langevin equation. Dynamic properties were studied through two important physical quantities, which are MSD and VACF. This equation was solved exactly with an appropriate choice of the memory-function, whose solution is expressed in terms of Mittag-Leffler functions.

For Pickering emulsions, it was demonstrated that the diffusion of a given oil-droplet is normal, at short-time, and subdiffusive, at large-time, with an anomalous exponent between 0 and 1 , and the theoretical predictions were found to be in perfect agreement with very recent MD simulations ${ }^{[10]}$.

An important question to ask is how particles that are embeded on the surface of a crowded system, can diffuse in time. Such a question was ensured in the case of fluid or polymerized membranes ${ }^{[69]}$ and the oil-droplets of a Pickering emulsion ${ }^{[70]}$. The main result is that, the random walker executes a subdiffusion, at short-time, followed by a saturation regime, at infinite time.

Finally, it will be interesting to extend these extensive dynamic studies to the situation where the diffusing particles have arbitrary shapes and forms. 


\section{Acknowledgements:}

I am much indebted to Professors D. Durand and L. Benyahia for helpful discussions. I would like to thank the Le Mans University for their kind hospitality during my regular visits.

\section{REFERENCES}

1. A.Blumen, J.Klafter, G.Zumofen, in Optical Spectroscopy of Glasses, edited by I.Zschokke, Reidel, Dordrecht (1986)

2. S.Havlin, D.Ben-Avraham,Adv. Phys., vol.36, p 187(1987)

3. J.-P.Bouchaud, A.Georges, Phys. Rep., vol.195, p 127 (1990)

4. See, for instance, D.Nualart, Fractional Brownian Motion: Stochastic Calculus and Applications, Proceedings of the International Congress of Mathematicians, edited by European Mathematical Society, Madrid, Spain (2006)

5. S.Stachura, G.R.Kneller,Molecular Simulation, vol.40, p 245 (2014)

6. H.Scher, M.Lax, Phys. Rev. B, vol.7, p 4491 (1973); Phys. Rev. B, vol.7, p 4502 (1973); H.Scher, E.Montroll, Phys. Rev. B, vol.12, p 2455 (1975)

7. Q.Gu, E.A.Schiff, S.Grebner, R.Schwartz, Phys. Rev. Lett., vol.76, p 3196 (1996)

8. H.-P.Müller, R.Kimmich, J.Weis, Phys. Rev. E, vol.54, p 5278 (1996); A.Klemm, H.-P.Müller, R.Kimmich, Phys. Rev. E, vol.55, p 4413 (1997)

9. F.Amblard, A.C.Maggs, B.Yurke, A.N.Pargellis, S.Leibler, Phys. Rev. Lett., vol.77, p 4470 (1996)

10. E.R.Weeks, H.L.Swinney, Phys. Rev. E, vol.57, p 4915 (1998)

11. G.Zumofen, J.Klafter, A.Blumen, J. Stat. Phys., vol.65, p 991 (1991)

12. L.F.Richardson, Proc. R. Soc. LondonSer. A, vol.110, p 709 (1926); G.K.Batchelor, Q.J.R. Meteorol. Soc., vol.76, p 133 (1950); P.Tabeling, A.E.Hansen, J.Paret, in Chaos, Kinetics and Nonlinear Dynamics in Fluids and Plasmas, edited by G.M.Zaslavsky and S.Benkadda, Springer-Verlag, Berlin (1998)

13. S.C.Kou, X.S.Xie, Phys. Rev. Lett., vol.93, p 180603 (2004)

14. N.Pottier, A.Mauger, Physica A, vol.282, p 77 (2000)

15. E.Lutz, Phys. Rev. E, vol.64, p 051106 (2001)

16. N.Pottier, Physica A, vol.317, p 371(2003)

17. P.Debnath, W.Min, X.S.Xie, B.J.Cherayila, J. Chem. Phys., vol.123, p 204903 (2005)

18. I.Goychuk, P.Hänggi, Phys. Rev. Lett., vol.99, p 200601(2007); I.Goychuk, Phys. Rev. E, vol.80, p 046125(2009)

19. S.Burov, E.Barkai, Phys. Rev. E, vol.78, p 031112(2008)

20. R.Kubo, Rep. Progr. Phys., vol.29, p 255 (1966); also, R.Kubo, M.Toda, N.Hashitsume, Statistical Physics II: Nonequilibrium Statistical Mechanics, Vol. 31, Springer Science \& Business Media (2012)

21. R.Metzler, E.Barkai, J.Klafter,Phys. Rev. Lett., vol.82, p 3563 (1999)

22. R.Metzler, J.Klafter, J. Phys. A: Math. Gen., vol.37, p R16 (2004)

23. T.Sandev, R.Metzler, Z.Tomovski, J. Phys. A: Math. Theor., vol.44, p 255203(2011)

24. R.Metzler, J.Klafter, Phys. Rep., vol.339, p 1 (2000)

25. R.Metzler, Phys. Rev. E, vol.62, p 6233(2000)

26. C.H.Eab, S.C.Lim, Physica A, vol.389, p 2510 (2010)

27. S.C.Lim, L.P.Teo, J. Stat. Mech., vol.42, p 065208(2009)

28. F.Mainardi, P.Pironi, Extracta Math., vol.10, p 140 (1996)

29. G.Mittag-Leffler, C. R. Acad. Sci., Paris, vol.137, p 554 (1903)

30. A.Wiman, Acta Math., vol.29, p 191 (1905)

31. R.P.Agarwal, C. R. Acad. Sci., Paris, vol.236, p 2031(1953)

32. P.Humbert, C. R. Acad. Sci., Paris,vol.236, p 1467 (1953)

33. P.Humbert, R.P.Agarwal, Bull. Sci. Math., vol.2, p 180 (1953) 
34. T.R.Prabhakar, Yokohama Math. J., vol.19, p 7 (1971)

35. H.M.Srivastava, Z.Tomovski, Appl. Math. Comput., vol.211, p 198 (2009)

36. A.K.Shukla, J.C.Prajapati, J. Math. Anal. Appl., vol.336, p 797 (2007)

37. R.K.Saxena, A.M.Mathai, H.J.Haubold, Astrophys. Space Sci., vol.209, p 299 (2004)

38. R.Hilfer, Applications of Fractional Calculus in Physics, World Scientific Publishing Company, Singapore (2000)

39. R.Hilfer, J.Seybold, Spec. Funct., vol.17, p 637 (2006)

40. I.Podlubny, Fractional Differential Equations, Academic Press, San Diego (1999)

41. E.Capelas de Oliveira, F.Mainardi, J.Vaz Jr., Eur. Phys. J. Spec. Top., vol.193, p 161 (2011)

42. T.Sandev, Z.Tomovski, J.L.A.Dubbeldam, Physica A, vol.390, p 3627(2011)

43. W.Ramsden, Proc. Royal Soc. (London), vol.72, p 156 (1903)

44. S.U.Pickering, J. Chem. Soc. Transactions, vol.91, p 2001 (1907)

45. M.Badia, S.El-Moudny, M.Benhamou, M.El Ossmani, J. Mol. Liquids, vol.240, p 1 (2017)

46. B.J.Alder, T.E.Wainwright, J. Chem. Phys., vol.27, p 1208 (1957)

47. R.Aveyard, B.P.Binks, J.H.Clint, Adv.Coll.Inter.Sci., vol.100-102, p 503 (2003)

48. B.P.Binks, Curr. Opin. Coll. Inter. Sci., vol.7, p 21 (2002)

49. B.P.Binks, T.S.Horozov, Colloidal Particles at Liquid Interfaces, Cambridge University Press, Cambridge (2006)

50. Also, see a recent nice review by Y.Chevalier, M.-A.Bolzinger, Coll. Surf. A: Physicochemical and Engineering Aspects, vol.439, p 23 (2013)

51. N.P.Pardhy, B.M.Budhlall, Langmuir, vol.26, p 13130 (2010)

52. J.H.Chen, C.Y.Cheng, W.Y.Chiu, C.F.Lee, N.Y.Liang, Eur. Polym. J., vol.44, p 3271 (2008)

53. P.J.Colver, C.A.Colard, S.A.Bon, J. Am. Chem. Soc., vol.130, p 16850 (2008)

54. C.Wang, C.Zhang, Y.Li, Y.Chen, Z.Tong, Reactive and Functional Polymers, vol 69, p 750 (2009)

55. K.Zhang, W.Wu, H.Meng, K.Guo, J.F.Chen, Powder Technology, vol.190, 393 (2009)

56. H.Ma, M.Luo, S.Sanyal, K.Rege, L.L.Dai, Materials, vol.3, p 1186(2010)

57. G.Yin, Z.Zheng, H.Wang, Q.Du, J. Coll. Inter. Sci., vol.361, p 456 (2011)

58. M.Zhang, T.H.Ngo, N.I.Rabiah, T.P.Otanicar, P.E.Phelan, R.Swaminathan, L.L.Dai, Langmuir, vol.30, p 75 (2013)

59. L.Hao, C.Wang, Z.Tong, Journal of Controlled Release, vol.172, p 19 (2013)

60. A.K.Dyab, H.A.Al-Lohedan, H.A.Essawy, A.I.A.El-Mageed, F.Taha, J. Saudi Chem. Soc., vol.18, p 610 (2014)

61. J.Ji, S.Shu, F.Wang, Z.Li, J.Liu, Y.Song, Y.Jia, Nanoscale Research Letters, vol.9, p 1 (2014)

62. A.D.Dinsmore, M.F.Hsu, M.G.Nikolaides, M.Marquez, A.R.Bausch, D.A.Weitz, Science, vol.298, p 1006 (2002)

63. T.Chen, P.J.Colver, S.A.Bon, Advanced Materials, vol.19, p 2286 (2007)

64. W.Chen, X.Liu, Y.Liu, H.I.Kim, Coll. Polym. Sci., vol.288, p 1393 (2010)

65. S.El-Moudny, M.Badia, M.Benhamou, J. Mol. Liquids, vol.225, p 174 (2017)

66. R.Zwanzig, Nonequilibrium Statistical Mechanics, Oxford University Press -2001); also, R.Zwanzig, Statistical Mechanics of Irreversibility, Vol. 3 of Lectures in Theoretical Physics, Interscience, New York (1961)

67. M.P.Allen, D.J.Tildesley, Computer Simulation of Liquids, Oxford University Press (1989)

68. H.J.Berendsen, J.P.M.Postma, W.F.van Gunsteren, A.R.H.J.DiNola, J.R.Haak, J. Chem. Phys., vol.81, p 3684 (1984)

69. S.Komura, A.Baumgärtner, J. Phys. France, vol.51, p 2395 (1990)

70. M.Benhamou, S.El-Moudny, International Journal of Nanomaterials, Nanotechnology and Nanomedicine submitted, vol. 3, p 057 (2017) 
Important: Articles are published under the responsability of authors, in particular concerning the respect of copyrights. Readers are aware that the contents of published articles may involve hazardous experiments if reproduced; the reproduction of experimental procedures described in articles is under the responsability of readers and their own analysis of potential danger.

\section{Reprint freely distributable - Open access article}

Materials and Devices is an Open Access journal which publishes original, and peer-reviewed papers accessible only via internet, freely for all. Your published article can be freely downloaded, and self archiving of your paper is allowed and encouraged!

We apply « the principles of transparency and best practice in scholarly publishing » as defined by the Committee on Publication Ethics (COPE), the Directory of Open Access Journals (DOAJ), and the Open Access Scholarly Publishers Organization (OASPA). The journal has thus been worked out in such a way as complying with the requirements issued by OASPA and DOAJ in order to apply to these organizations soon.

Copyright on any article in Materials and Devices is retained by the author(s) under the Creative Commons

(Attribution-NonCommercial-NoDerivatives 4.0 International (CC BY-NC-ND 4.0)), which is

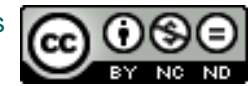
favourable to authors.

Aims and Scope of the journal : the topics covered by the journal are wide, Materials and Devices aims at publishing papers on all aspects related to materials (including experimental techniques and methods), and devices in a wide sense provided they integrate specific materials. Works in relation with sustainable development are welcome. The journal publishes several types of papers : A: regular papers, L : short papers, $R$ : review papers, $T$ : technical papers, Ur : Unexpected and « negative » results, Conf: conference papers.

(see details in the site of the journal: http://materialsanddevices.co-ac.com)

We want to maintain Materials and Devices Open Access and free of charge thanks to volunteerism, the journal is managed by scientists for science! You are welcome if you desire to join the team!

Advertising in our pages helps us! Companies selling scientific equipments and technologies are particularly relevant for ads in several places to inform about their products (in article pages as below, journal site, published volumes pages, ...). Corporate sponsorship is also welcome!

Feel free to contact us! contact@co-ac.com 